\title{
Neuronal activity (c-Fos) delineating interactions of the cerebral cortex and basal ganglia
}

\author{
Mei-Hong Qiu' ${ }^{*}$, Michael C. Chen ${ }^{2}$, Zhi-Li Huang ${ }^{1,3,4}$ * and Jun Lu ${ }^{2}$ * \\ 'State Key Laboratory of Medical Neurobiology and Department of Neurobiology, School of Basic Medical Science, Fudan University, Shanghai, China \\ ${ }^{2}$ Neurology Department and Division of Sleep Medicine, Beth Israel Deaconess Medical Center and Harvard Medical School, Boston, MA, USA \\ ${ }^{3}$ Department of Pharmacology, School of Basic Medical Science, Fudan University, Shanghai, China \\ ${ }^{4}$ Institute of Brain Science, Fudan University, Shanghai, China
}

\section{Edited by:}

Yun-Qing Li, The Fourth Military

Medical University, China

\section{Reviewed by:}

Michael Lazarus, University of Tsukuba, Japan

Yi-Ping Hou, Lanzhou University, China

\section{*Correspondence:}

Mei-Hong Qiu, State key Laboratory

of Medical Neurobiology and

Department of Neurobiology, School

of Basic Medical Science, Fudan

University, Room 705, Zhi Dao

Building, 138 Yixueyuan Road,

Shanghai 200032, China

e-mail:mhqiu@shmu.edu.cn;

Zhi-Li Huang, Department of

Pharmacology, School of Basic

Medical Science, Fudan University,

Room 107, Building 7, 138 Yixueyuan

Road, Shanghai 200032, China

e-mail: huangzl@fudan.edu.cn;

Jun Lu, Neurology Department and

Division of Sleep Medicine, Beth

Israel Deaconess Medical Center and

Harvard Medical School, 707 CLS,

Blackfan Circle \#3, Boston, MA 02115,

USA

e-mail: jlu@bidmc.harvard.edu
The cerebral cortex and basal ganglia (BG) form a neural circuit that is disrupted in disorders such as Parkinson's disease. We found that neuronal activity (c-Fos) in the BG followed cortical activity, i.e., high in arousal state and low in sleep state. To determine if cortical activity is necessary for BG activity, we administered atropine to rats to induce a dissociative state resulting in slow-wave electroencephalography but hyperactive motor behaviors. Atropine blocked c-Fos expression in the cortex and BG, despite high c-Fos expression in the sub-cortical arousal neuronal groups and thalamus, indicating that cortical activity is required for BG activation. To identify which glutamate receptors in the BG that mediate cortical inputs, we injected ketamine [N-methyl-D-aspartate (NMDA) receptor antagonist] and 6-cyano-nitroquinoxaline-2, 3-dione (CNOX, a non-NMDA receptor antagonist). Systemic ketamine and $\mathrm{CNOX}$ administration revealed that NMDA receptors mediated subthalamic nucleus (STN) input to internal globus pallidus (GPi) and substantia nigra pars reticulata (SNr), while non-NMDA receptor mediated cortical input to the STN. Both types of glutamate receptors were involved in mediating cortical input to the striatum. Dorsal striatal (caudoputamen, CPu) dopamine depletion by 6-hydroxydopamine resulted in reduced activity of the $\mathrm{CPu}$, globus pallidus externa (GPe), and STN but increased activity of the GPi, SNr, and putative layer $\mathrm{V}$ neurons in the motor cortex. Our results reveal that the cortical activity is necessary for BG activity and clarifies the pathways and properties of the BG-cortical network and their putative role in the pathophysiology of BG disorders.

Keywords: cerebral cortex, basal ganglia, arousal, atropine, 6-hydroxydopamine, c-Fos, rat

\section{INTRODUCTION}

The cerebral cortex and basal ganglia (BG), two of the largest forebrain structures, form a dynamic interactive network. The cerebral cortex, along with the thalamus and subcortical systems, sends signals to the BG. The BG in turn, with modulation from midbrain dopaminergic system, integrates and processes this cortical information for output, back to the cerebral cortex to shape cortical activity and ultimately affect cortical functions such as motor behavior (Yelnik, 2008). This reciprocal regulation is significantly altered after BG dysfunction, as in neurological disorders such as Parkinson's disease and Huntington's disease. Given the complex interconnections within the subunits of the $\mathrm{BG}$, and between the BG and cerebral cortex, it is critical to clarify how the cerebral cortex and BG interact and how this interaction is shaped by subcortical modulation, especially by dopamine.

Classically, BG and cerebral cortex networks have been organized into direct, indirect, and hyper-direct pathways. Dopamine $D_{1}$ and $D_{2}$ receptor containing medium spiny neurons in the striatum (dorsal part, or caudate-putamen, $\mathrm{CPu}$ ) receive glutamatergic cortical input and project to the internal segment of the globus pallidus (GPi)/substantia nigra pars reticulata (SNr; direct pathway) and external segment of globus pallidus (GPe; indirect pathway). The GPe and SNr/GPi have GABAergic projections to the mediodorsal thalamus (MD) and the motor thalamus, respectively (Nauta, 1979; Parent and De Bellefeuille, 1983; Haber et al., 1985), which innervate the medial prefrontal, motor, and somatosensory cortices, respectively. In addition, the glutamatergic subthalamic nucleus (STN) also receives cortical inputs (hyper-direct pathway) and in turn projects primarily to the GPe, GPi, and SNr. Midbrain dopaminergic neurons provide the major subcortical input to the $\mathrm{BG}$, especially the striatum. These projections form the core of the classical model of BG organization that influences current thinking about BG function and dysfunction, as in Parkinson's and Huntington's disease.

Single-unit recordings show that neurons in the BG, i.e., the striatum, GPe, $\mathrm{SNr}$, and STN, are more active during wake and rapid eye movement (REM) sleep and less active and synchronized to cortex electroencephalography (EEG) in slow-wave sleep or in an anesthetized state (Detari et al., 1987; Magill et al., 2000; 
Urbain et al., 2000; Mahon et al., 2006). Consistent with this evidence, we have previously demonstrated that c-Fos expression (a marker of neuronal activity) in BG subunits is higher during spontaneous wake and methamphetamine-induced arousal, than during sleep (Vetrivelan et al., 2010). These studies suggest that excitatory cortical input to the BG may regulate BG activity. Given that the BG also receives subcortical inputs and projects to the cortex, it is unclear whether the cortical activity is necessary for BG activity.

We examined BG-cortex activity using c-Fos expression after wakefulness, systemic atropine injection, and after blocking NMDA and non-NMDA receptors. Our results suggest that cortical neuronal activity is necessary for BG neuronal activity and that the cortex shapes BG activity via two pathways: NMDA/non-NMDA receptor-mediated cortical inputs to the striatum, and non-NMDA receptor-mediated cortical inputs to the STN. We then examined if cortical activity is affected by BG activity alterations caused by dopamine depletion. Striatal dopamine depletion resulted in hyperactivity of the GPi, SNr, and motor cortex, as well as hypoactivity of the dorsal striatum, GPe, and STN, but only during high cortical activity wakefulness. These changes in BG and cortical activity during dopamine depletion may underlie the various motor and non-motor symptoms of BG disorders, including Parkinson's disease.

\section{MATERIALS AND METHODS ANIMALS}

Pathogen-free, adult, male Sprague-Dawley rats (275-300 g, Harlan) were individually housed and had ad libitum access to food and water. All animals were housed under light-controlled conditions (12 h light starting at 07:00 AM, $100 \mathrm{lux}$ ) in an isolated ventilated chamber maintained at $20-22^{\circ} \mathrm{C}$, All protocols were approved by the Institutional Animal Care and Use Committees of Beth Israel Deaconess Medical Center and the experiments were carried out in accordance with the Guidelines laid down by the $\mathrm{NIH}$ in the US regarding the care and use of animals for experimental procedures. Every effort was made to minimize the number of animals used and any pain and discomfort experienced by the subjects.

\section{ACTIVE-WAKE (AW) CONDITION}

Four cages were aligned without covers in a hood, after saline or drug injection (see below), rats were allowed to freely move over and between cages. Under this social and motor-active condition, rats will remain awake and highly active for at least $4 \mathrm{~h}$, as previously reported (Gompf et al., 2010). Rats were perfused $2 \mathrm{~h}$ after AW condition. The sleep control group consisted of animals that were perfused $2 \mathrm{~h}$ after saline injections around 9:00-10:00 AM. The perfused brains were processed for histology.

\section{6-HYDROXYDOPAMINE (6-OHDA) INJECTIONS}

Animals were anesthetized with chloral hydrate $(350 \mathrm{mg} / \mathrm{kg})$ and received unilateral 6-OHDA $(6 \%, 90 \mathrm{nl}$, Sigma) injections into the ventral GPe (coordinates: $\mathrm{AP}=0.8 \mathrm{~mm}, \mathrm{ML}=3.0 \mathrm{~mm}$, $\mathrm{DV}=7.0 \mathrm{~mm}$ ) through a glass pipette by air pressure. Animals were allowed to recover for 2 weeks prior to experiments and perfusion. This method of dopamine depletion has been confirmed in previous reports (Abedi et al., 2013).

\section{DRUG ADMINISTRATION SCIENCE AND ENGINEERING REFERENCES}

Atropine (100 mg/kg, Sigma), Ketamine (35 mg/kg, Sigma), and CNQX (3.0 mg/kg, Sigma) were dissolved in saline immediately before use and administered via intraperitoneal injection between 9:00 and 10:00 AM.

\section{EEG/EMG RECORDING}

We recorded EEG/EMG and video in two rats received atropine injections for $2 \mathrm{~h}$, to confirm that atropine produces slow wave EEG and locomotion with high EMG. The surgery procedure and EEG analyses have been described in many of our previous publications (Lu et al., 2006; Vetrivelan et al., 2009; Qiu et al., 2010).

\section{IMMUNOHISTOCHEMISTRY}

Animals were deeply anesthetized by chloral hydrate $(500 \mathrm{mg} / \mathrm{kg})$ and perfused with $50 \mathrm{ml}$ saline followed by $500 \mathrm{ml} 10 \%$ formalin through the heart. The brains were removed, post-fixed for $4 \mathrm{~h}$ in $10 \%$ formalin, and equilibrated in $20 \%$ sucrose in phosphate-buffered saline (PBS) overnight. The brains were frozen and sectioned on a freezing microtome at $40 \mu \mathrm{m}$ into four series. Sections were washed in 0.1 M PBS, pH 7.4 (two changes) and then incubated in the primary antiserum (c-Fos, 1:50 K, AB5, Oncogene; choline acetyltransferase, ChAT, 1:500, AB144, Chemicon; tyrosine hydroxylase, TH, 1:20K, \#22941, Diasorin) for $24 \mathrm{~h}$ at room temperature. On the second day, the sections were washed in PBS and incubated in biotinylated secondary antiserum (against appropriate species IgG, 1:1,000 in PBS) for $1 \mathrm{~h}$, followed by a 1:1000 dilution of avidin-biotinperoxidase (Vector Laboratories, Burlingame, CA, USA) for $1 \mathrm{~h}$ at room temperature. The peroxidase reaction was visualized with $0.05 \%$ 3,3-diaminobenzidine tetrahydrochloride (DAB, Sigma) in PBS and $0.01 \% \mathrm{H}_{2} \mathrm{O}_{2}$. The sections were stained brown with DAB only or black by adding $0.05 \%$ cobalt chloride and $0.01 \%$ nickel ammonium sulfate to the DAB solutions. c-Fos was labeled black and TH and ChAT labeled brown to enable distinct staining within the same section. After terminating the reaction by PBS-azide, sections were mounted, dehydrated and cover slipped. As controls, adjacent sections were incubated without the primary antibody to confirm that no non-specific staining had occurred.

\section{CELl COUNTING}

c-Fos positive neurons were counted in the BG of three adjacent sections that contain BG structures. For the striatum, the $2.0 \mathrm{~mm} \times 2.0 \mathrm{~mm}$ counting box was placed in the center of the dorsomedial striatum. For c-Fos counting in M1/M2 cortex, only black-stained large neurons (likely projecting pyramidal neurons) were counted in the entire M1/M2 cortex. For other BG subunits, we counted all c-Fos positive neurons in the entire region of three adjacent sections. The c-Fos counts represented by average counting per section per side were used to construct Tables 1 and 2. We used, paired $T$-test for count differences. 
Table 1 | c-Fos expression in BG and cortex.

\begin{tabular}{|c|c|c|c|c|c|}
\hline & Sleep & AW & Atropine & Ketamine & CNOX \\
\hline Striatum & $15.6 \pm 7.0$ & $179.0 \pm 15.2$ & $23.5 \pm 5.7^{* *}$ & $75.7 \pm 13.4^{* / \# \#}$ & $134.3 \pm 11.4^{* / \# \#}$ \\
\hline GPi & $2.2 \pm 0.9$ & $24.2 \pm 5.5$ & $2.0 \pm 0.7^{* *}$ & $4.2 \pm 1.3^{* *}$ & $3.43 \pm 0.9^{* *}$ \\
\hline STN & $2.2 \pm 1.6$ & $97.8 \pm 9.0$ & $10.6 \pm 3.2^{* * / \#}$ & $85.2 \pm 8.2^{\# \#}$ & $6.2 \pm 1.9^{* *}$ \\
\hline $\mathrm{SNr}$ & $1.8 \pm 1.3$ & $23.6 \pm 4.3$ & $1.8 \pm 1.4^{* *}$ & $1.9 \pm 1.3^{* *}$ & $2.2 \pm 1.9^{* *}$ \\
\hline Cortex & Inactive & Active & Inactive & Active & Active \\
\hline Thalamus & Inactive & Active & Active & Active & Active \\
\hline
\end{tabular}

LC: " $+"=25 c$-Fos positive neurons. All c-Fos counting is presented by number/section/side. The difference ${ }^{*} p<0.05, * * p<0.01$ of T-test is compared to the AW. The difference of $\# p<0.05, \# \# p<0.01$ is compared to sleep condition.

Table 2 | c-Fos expression in intact $(\mathrm{DA}+/+$ ) vs. DA depleted side $\left(\mathrm{DA}-\mathrm{I}-\right.$ ) in AW*p $<0.05,{ }^{*} \boldsymbol{p}<0.01$.

\begin{tabular}{lcl}
\hline & DA+l+ & DA-I- \\
\hline Striatum & $185.3 \pm 16.2$ & $95.4 \pm 20.2^{*}$ \\
GPe & $55.2 \pm 8.8$ & $32.5 \pm 13.1^{*}$ \\
GPi & $22.5 \pm 6.8$ & $75.9 \pm 19.2^{* *}$ \\
STN & $105.8 \pm 12.6$ & $64.7 \pm 8.5^{*}$ \\
SNr & $26.1 \pm 5.5$ & $75.3 \pm 12.2^{* *}$ \\
M1-M2 & $15.0 \pm 10.1$ & $38.7 \pm 15.8^{* *}$ \\
\hline
\end{tabular}

\section{RESULTS}

\section{CORTICAL ACTIVITY PREDICTS BG ACTIVITY}

Although we have previously shown c-Fos expression in the BG during spontaneous and methamphetamine-induced wake (Vetrivelan et al., 2010), we sought to determine c-Fos expression in the BG during an active-wake (AW) condition, in which rats remain spontaneous active awake in the sleep period (8:00 AM12:00 PM) and engage in social and motor behavior (see Materials and Methods). To determine BG activity in the AW condition, we injected saline and perfused rats in two conditions: after $2 \mathrm{~h}$ of sleep $(N=5)$ or after $2 \mathrm{~h}$ of AW $(N=5)$, during which animals had high locomotion and social interactions. Animals were perfused between 11:00 AM and 12:00 PM. The perfused brains were sectioned at $40 \mu \mathrm{m}$ in four series, and two series of sections each were double immunolabeled with c-Fos (black color) and TH (brown color), or c-Fos (black color) and ChAT (brown color), respectively.

Compared to the sleep condition, c-Fos expression was significantly higher in the cerebral cortex in AW rats, especially in the prefrontal and frontal, motor, and sensory cortices (Table 1; Figure 1A) and in all the subunits of the BG, i.e., the dorsal striatum (i.e., caudoputamen, $\mathrm{CPu}$ ), GPe, GPi, STN, and $\mathrm{SNr}$ (Table 1; Figures 1B-F). There was a distinct pattern of c-Fos expression in the dorsomedial part in the striatum that receives inputs from the premotor (M2) and primary motor (M1) cortices (Figure 1B). The medial part of the GPe, GPi, SNr, and STN also showed strong c-Fos expression (Table 1; Figures 1C-F). The pattern and amount of waking c-Fos expression were similar to that following methamphetamine injection but much higher

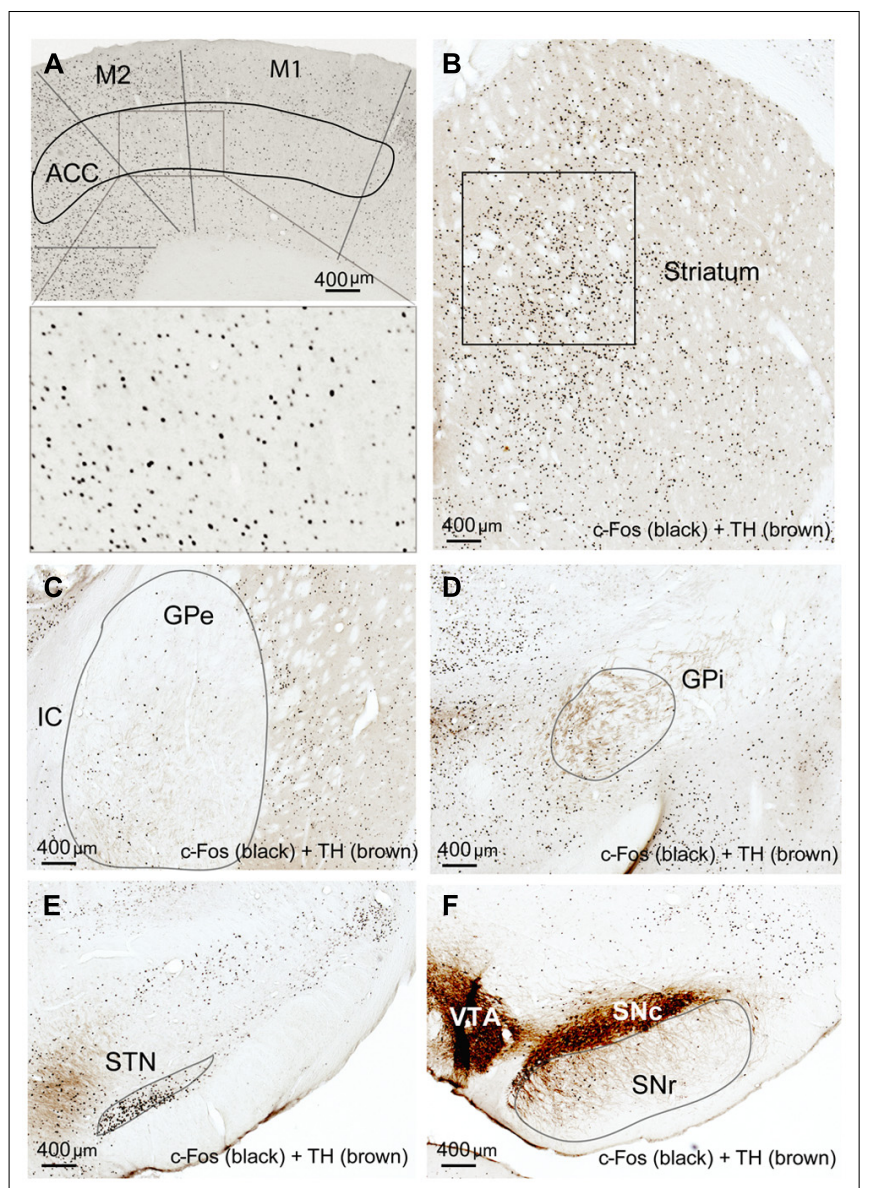

FIGURE 1 | Active-wake (AW) induces c-Fos expression in BG and cortex. High c-Fos expression is seen in the cerebral cortex (A), striatum (B), GPe (C), GPi (D), STN (E), and SNr (F) by AW condition. c-Fos active neurons are clustered in the medial part of the dorsal striatum, GPe, GPi, and SNr, which have strong interconnectivity with motor cortex. 

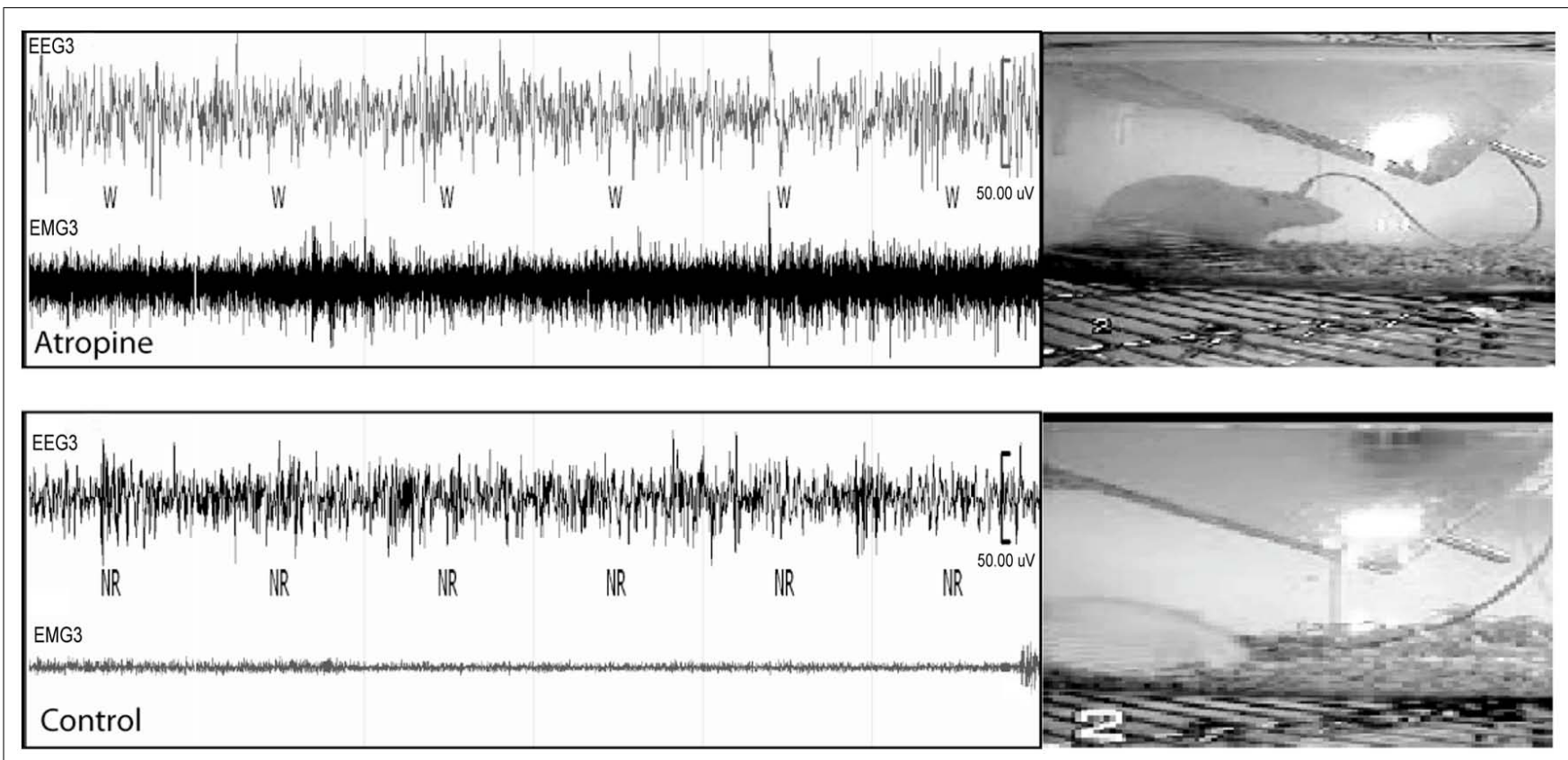

FIGURE 2 | Atropine induces slow-wave EEG and high EMG. In 60 s EEG/EMG and video recording trace, a rat shows high EMG, standing posture but sleep-like slow-wave EEG after atropine injection (upper panel), which is very different from normal sleep behavior with slow-wave EEG, low EMG, and sleep posture (lower panel). $W=$ wake, NR = NREM sleep.

than spontaneous wakefulness (Vetrivelan et al., 2010). These results indicate that BG neural activity is increased when cortical neuronal activity is increased and likewise decreased when cortical activity is decreased, as in the slow-wave EEG state like sleep.

\section{CORTICAL ACTIVITY IS NECESSARY FOR BG ACTIVITY}

To determine whether cortical activity is necessary for BG activity, we injected atropine, which induces a unique dissociative behavioral state with slow-wave EEG but heightened locomotion (Irmis, 1971; Davis et al., 2011). This "sleepwalking-like" state provides a unique opportunity to investigate whether cortical activity is necessary for BG activity. We injected atropine (100 $\mathrm{mg} / \mathrm{kg})$ at 9:00-10:00 AM and perfused the rats $2 \mathrm{~h}$ postinjection. The brains were sectioned, and the sections were immunolabeled with c-Fos and ChAT. Slow-wave EEG shown by atropine administration (Schaul et al., 1978) was confirmed by our EEG/EMG/video recording from two rats (Figure 2). Inconsistent with slow EEG, c-Fos expression in the cortex was very low (Figure 3A) and comparable to low c-Fos expression in the cortex in sleep condition (Table 1). In contrast, c-Fos was highly expressed in sub-cortical arousal regions such as the thalamus (intralaminar and midline region, Figure 6A), basal forebrain cholinergic neurons (Figure 6C), the tuberomammillary nucleus (TMN; Figure 6E), and the locus coeruleus (LC; Figure 6G), all resembling the pattern of c-Fos present in these areas during AW (Table 1). Along with low cortical activity, and despite high sub-cortical arousal activity, c-Fos expression in all BG subunits, following low cortical c-Fos expression, was low (Table 1; Figures 3B-F), resembling the sleep condition. These results

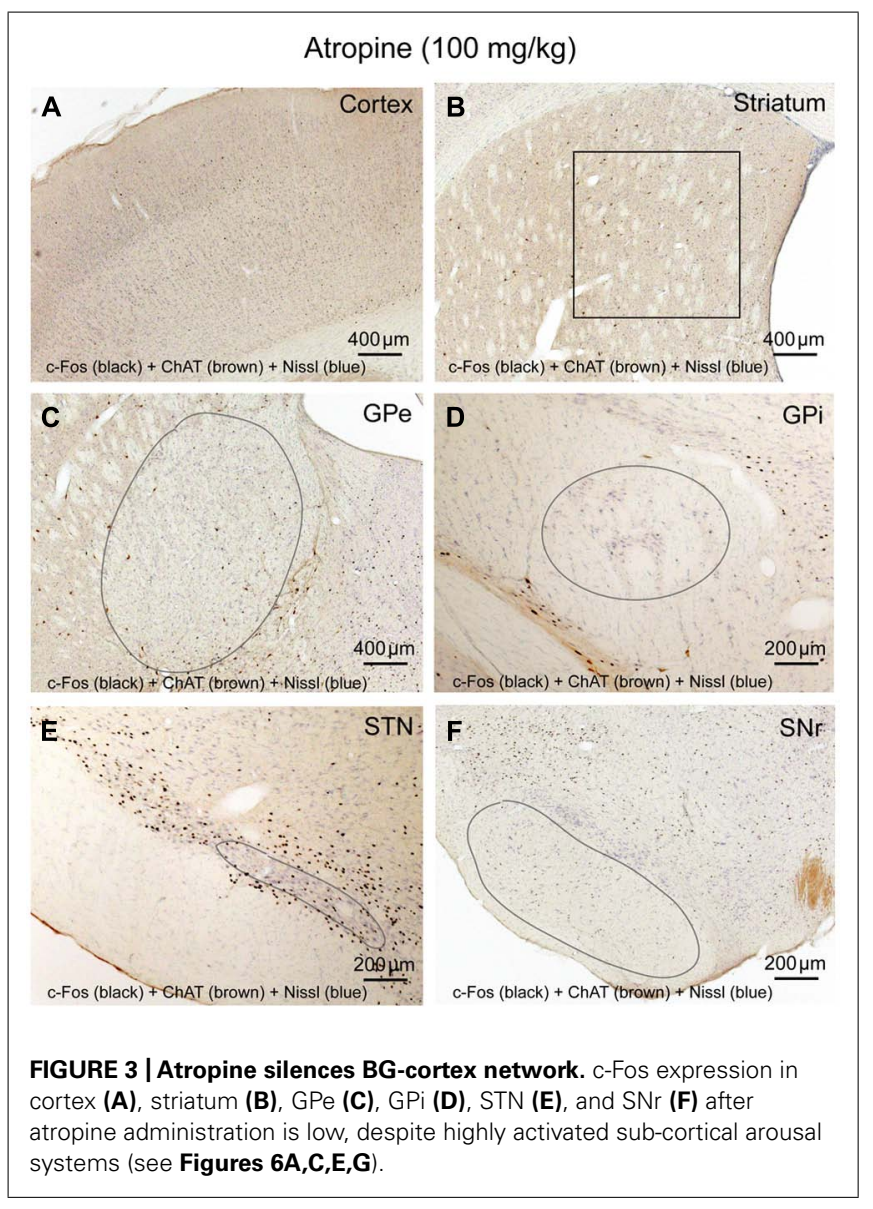




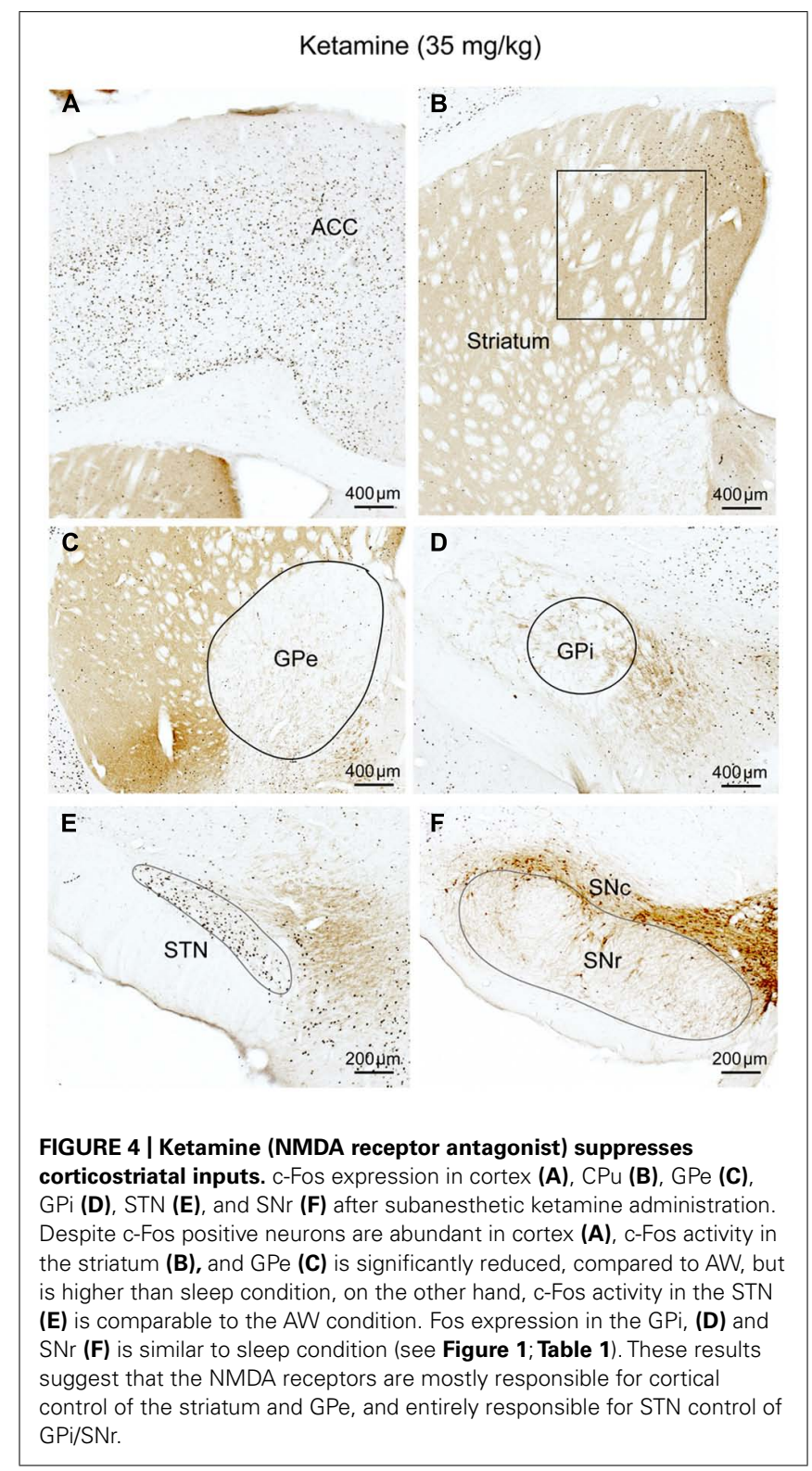

indicate that despite active subcortical arousal (locomotion) and active subcortical inputs to the BG, which was inactive when cortex was silenced. Thus cortical activity is necessary for BG neuronal activity.

\section{NMDA RECEPTORS MEDIATE CORTICAL INPUTS TO THE CPu}

We hypothesized that the cortex activates BG subunits by activating the striatum and the STN first via direct projections. Cortical input to the BG employs NMDA receptors as part of glutamatergic pathways (Ravenscroft and Brotchie, 2000). As these pathways potentially mediate cortical control over the BG, we examined whether NMDA and non-NMDA receptors are involved in both cortico-striatal and cortico-STN pathways, as well as pathways within the BG.

We administrated ketamine, a NMDA receptor antagonist, at a sub-anesthetic dose $(35 \mathrm{mg} / \mathrm{kg})$ to five rats in 9:00-10:00AM. As

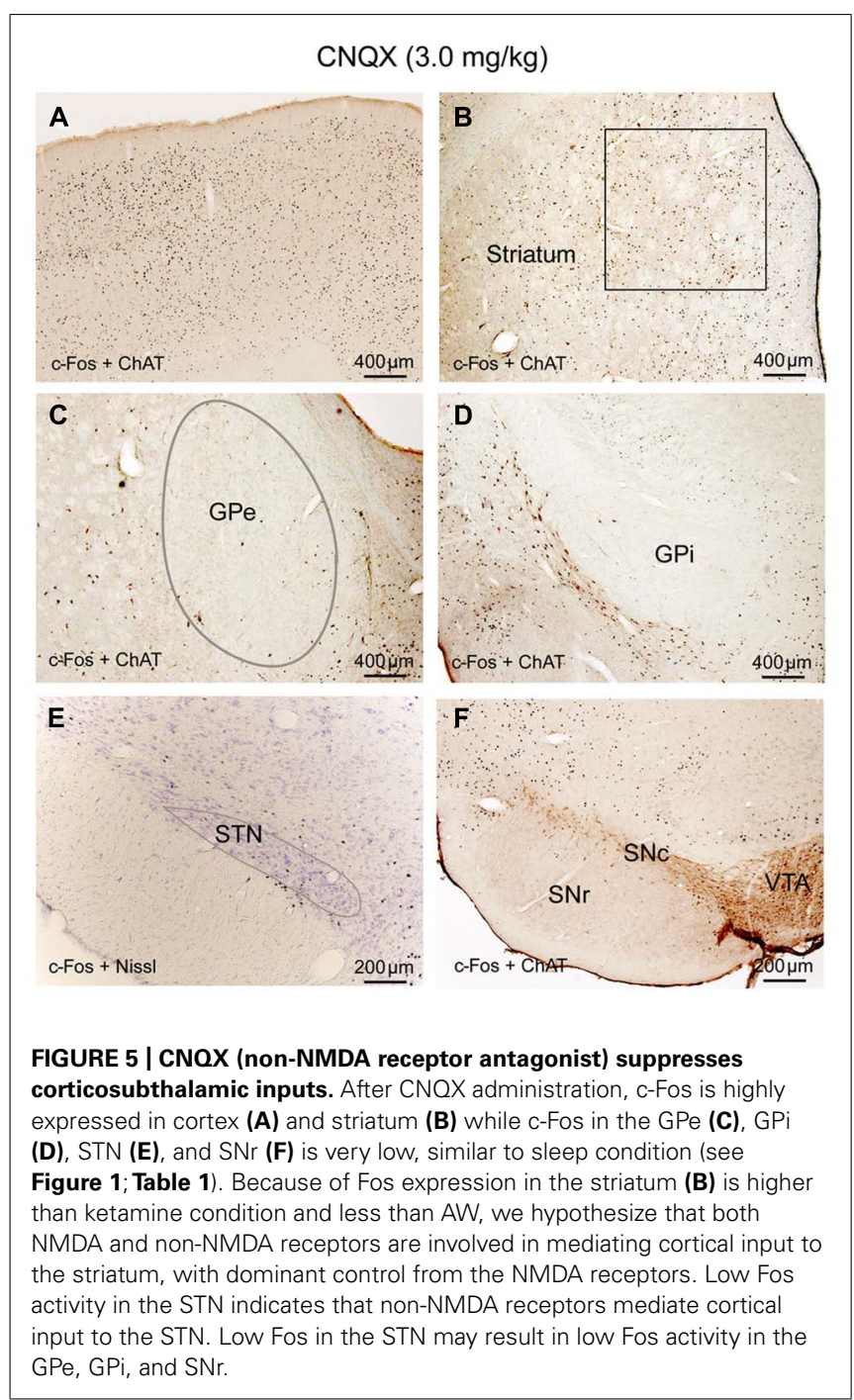

described before (Lu et al., 2008), animals were awake and active but rarely jumped to the other cages. Animals were perfused $2 \mathrm{~h}$ post-injection and the brains were sectioned and immunolabeled with c-Fos and TH. Ketamine at this dose increased locomotor behavior, wake-like EEG, and high c-Fos expression in arousal system structures including orexinergic neurons of the hypothalamus, LC, TMN, cortex, and thalamus, as previously demonstrated (Lu and Greco, 2006; Lu et al., 2008). Despite the increased c-Fos in the cortex, subcortical arousal systems, and thalamus system (Table 1; Figure 4A), as well as the presence of arousal behavior, c-Fos expression was lower in the CPu, GPi GPe, and $\mathrm{SNr}$ than during $\mathrm{AW}$, although c-Fos in the $\mathrm{CPu}$ and GPe was higher than during sleep or atropine condition (Figures 4B,F; Table 1). The STN showed high c-Fos expression, similar to the AW condition (Table 1; Figures $4 \mathrm{C}-\mathrm{E}$ ). These results suggest that NMDA receptors are involved in mediating cortical glutamate inputs to the $\mathrm{CPu}$ and STN glutamate inputs to the GPe, GPi, and SNr but not cortical glutamate inputs to the STN (Figure 9A). However, the finding that c-Fos in the $\mathrm{CPu}$ and GPe was higher than in atropine and sleep condition suggests 


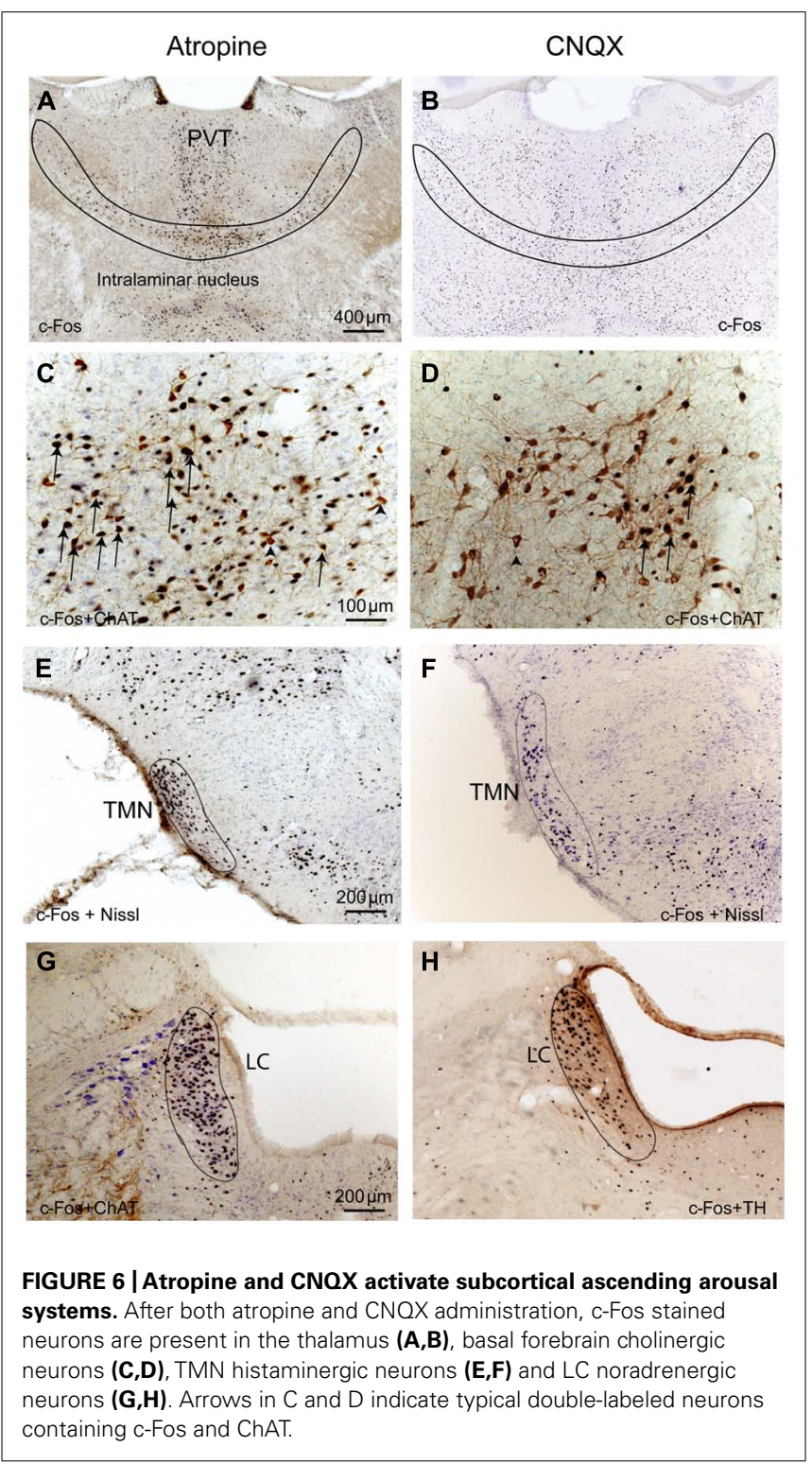

non-NMDA receptors may also be involved in inputs to these structures.

\section{NON-NMDA RECEPTORS MEDIATE CORTICAL INPUTS TO THE STN}

As NMDA receptors do not appear to entirely mediate cortical input to the striatum and STN input to the GPe, we examined the role of non-NMDA AMPA/kainate receptors in mediating glutamatergic cortical inputs to the BG. We injected CNQX, an AMPA/kainate receptor antagonist $(3.0 \mathrm{mg} / \mathrm{kg})$ at 9:00-10:00 AM in five rats and perfused those $2 \mathrm{~h}$ post-injection. The brains were sectioned and immunolabeled with c-Fos.

Until perfusion time, rats with CNQX injections were awake but appeared to have difficulty with locomotion; their body posture suggested low muscle tone (partial paralysis). c-Fos expression in the cortex and thalamus, as well as subcortical arousal systems including the TMN, LC, and BF were comparable to the ketamine and AW condition (Table 1; Figures 5A and 6B,D,F,H).

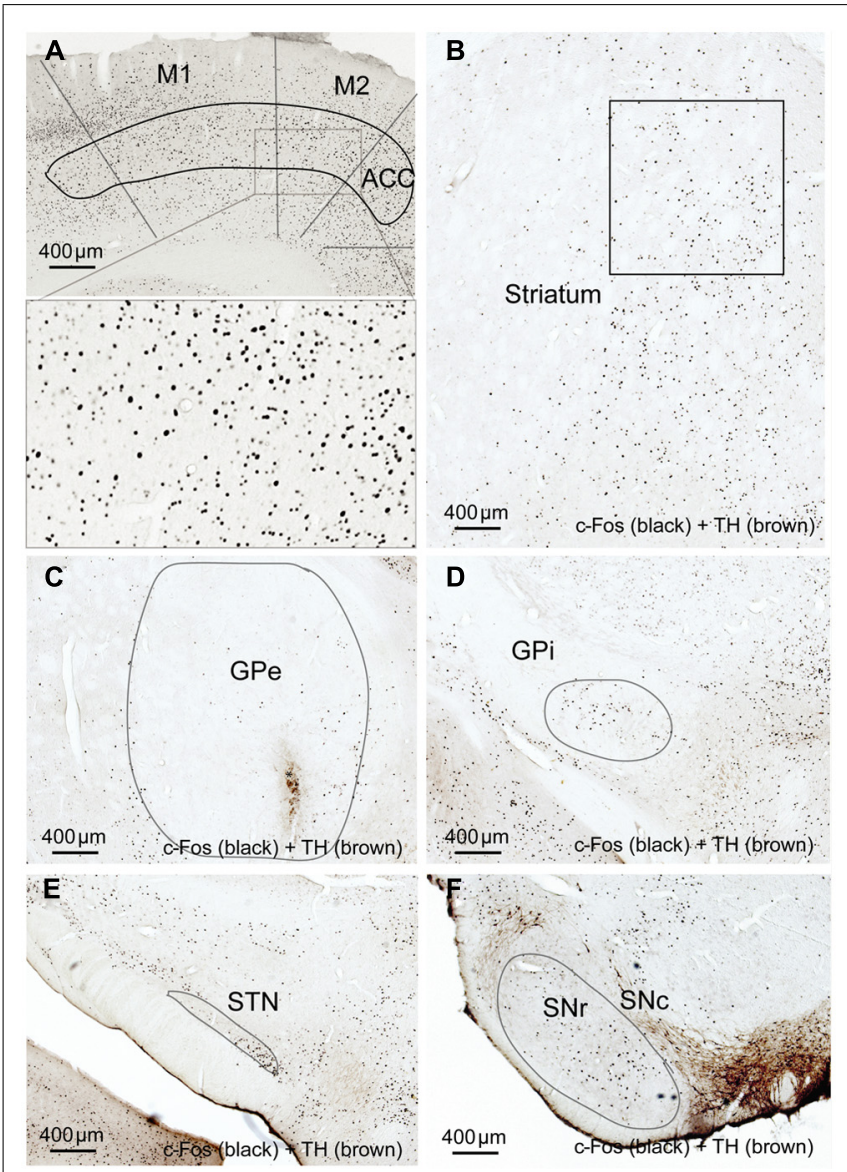

FIGURE 7 | Striatal dopamine depletion alters activity in cortex-BG. Compared to the unlesion side, c-Fos expression after dopamine depletion is increased in motor (M1-2) cortex (A), GPi (D) and SNr (F) but reduced in the striatum (B), GPe, (C) and STN (E). M1: primary motor cortex, M2: secondary motor cortex.

c-Fos in the striatum was higher than ketamine injection but less than the AW condition, c-Fos was absent in the GPe, GPi, SNr, and STN (Table 1; Figures 5B-F). These results, in conjunction with patterns of c-Fos expression during ketamine, suggest that non-NMDA (AMPA/kainate) receptors predominantly mediate cortical input to the STN, and they together with NMDA receptors mediate cortical input to the striatum, and STN input to the GPe (Figure 9A).

\section{DOPAMINE DEPLETION ALTERS BG AND CORTICAL ACTIVITY DURING ACTIVE WAKE}

The $\mathrm{CPu}$ receives dopaminergic input from the substantia nigra pars compacta $(\mathrm{SNc})$. To determine how this input regulates the $\mathrm{BG}$, we selectively destroyed SNc dopamine efferents to the BG by unilaterally injecting 6-OHDA into the ventral GPe in five rats. This lesion method has also been used and been verified recently in behavioral tests (Abedi et al., 2013). After the rats recovered from surgery, we examined the role of dopamine depletion on cortical input to the BG using two conditions: high cortical activity $(2 \mathrm{~h}$ of AW starting at 9:00-10:00 AM) and low cortical activity (2 h 


\section{A}
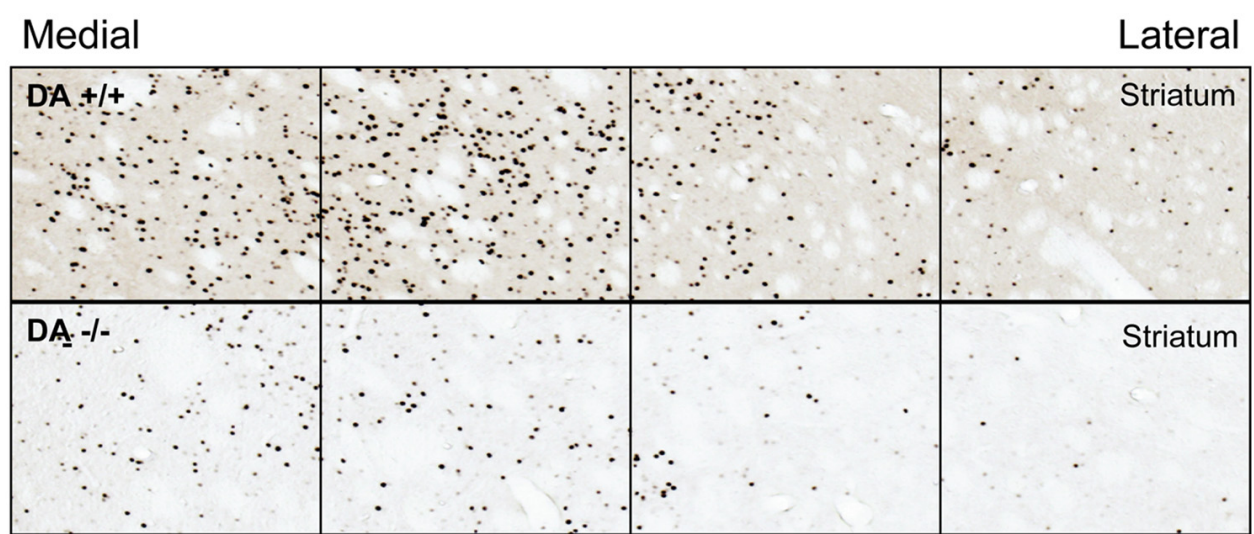

B

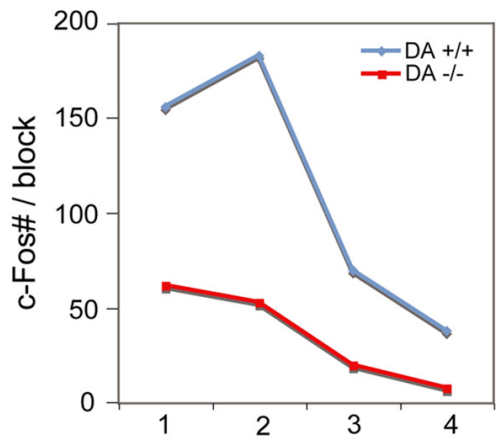

FIGURE 8 | Dopamine depletion evenly reduces c-Fos expression in the striatum. After unilateral striatal dopamine depletion, c-Fos expression in the striatum in AW is reduced in the lesion side, compared to the intact side
C

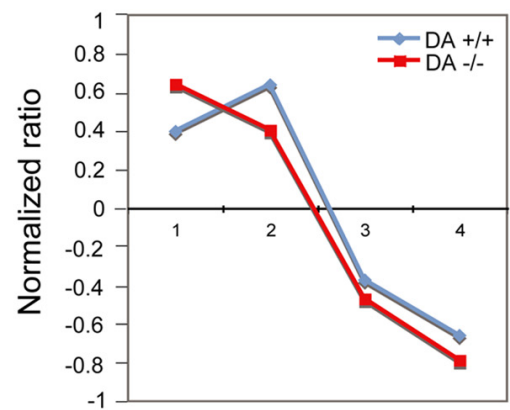

(A) c-Fos counts (B) and normalized value (count-average)/average (C) in four striatal counting areas indicate the reduction of c-Fos positive neurons by $6-O H D A$ is evenly distributed across the striatum. of sleep starting at 9:00-10:00 AM). After AW or sleep conditions, animals were perfused, and two series of sections were separately labeled with TH and c-Fos.

Tyrosine hydroxylase immunoreactivity showed selective loss of TH terminals in the dorsal striatum (caudate and putamen) of the 6-OHDA-injected animals, indicating dopamine depletion. The ventral striatum (nucleus accumbens and olfactory tubercle) and the dopaminergic neurons and terminals in the hypothalamus and amygdala were intact. In addition, LC TH-labeled neurons were intact.

In the high cortical activity AW condition, the number of large and intensely c-Fos-stained neurons in motor cortex M1 and M2 putative layer $\mathrm{V}$ neurons were higher in the lesioned side than the unlesioned side (Table 2; Figure 7A). Compared to the unlesioned side, dopamine depletion greatly reduced overall the number of $\mathrm{c}$-Fos stained neurons in the $\mathrm{CPu}$ (Table 2; Figure 7B). c-Fos reduction appeared to be even across the striatum (Figure 8). Likewise, the number of c-Fos stained neurons in the GPe and STN was substantially reduced (Figures 7C,E). In contrast, the number of c-Fos positive neurons in the GPi and $\mathrm{SNr}$ of the lesioned side was twice that of the unlesioned side (Table 2; Figures 7D,F).

In the low cortical activity sleep condition, we again observed loss of $\mathrm{TH}$ terminals in the dorsal striatum, with preserved $\mathrm{TH}$ expression in the ventral striatum, hypothalamus, amygdala, and LC. In contrast to the AW condition, in the low cortical activity sleep condition c-Fos expression in the BG in both lesioned and unlesioned sides was uniformly low, without any differences between the sides (data not shown). These results indicate that dopamine depletion alters BG activity during high cortical activity, but not during low cortical activity.

\section{DISCUSSION}

We have demonstrated that BG (CPu, GPe, GPi, SNr, and STN) neuronal activity was synchronized to, and driven by, cortical neuronal activity via two routes, corticostriatal and corticosubthalamic projections; the STN then activated the GPe, SNr, and GPi to complete the cortex-BG synchronization (Figure 9A). NMDA and non-NMDA receptors mediated cortical glutamatergic inputs to the striatum as well as STN inputs to the GPe; NMDA receptors mediated STN inputs to the SNr and GPi; nonNMDA receptors mediated cortical inputs to the STN (Figure 9A). Dopamine depletion reduced activity in the $\mathrm{CPu}, \mathrm{GPe}$, and STN but increased activity in the GPi, SNr, and motor cortex (Figure 9B). These changes were particularly prominent in the active arousal conditions.

We used c-Fos as a marker of neuronal activity, although there are some limitations with using c-Fos. For example certain 
A Neural pathways of cortex synchronizing BG
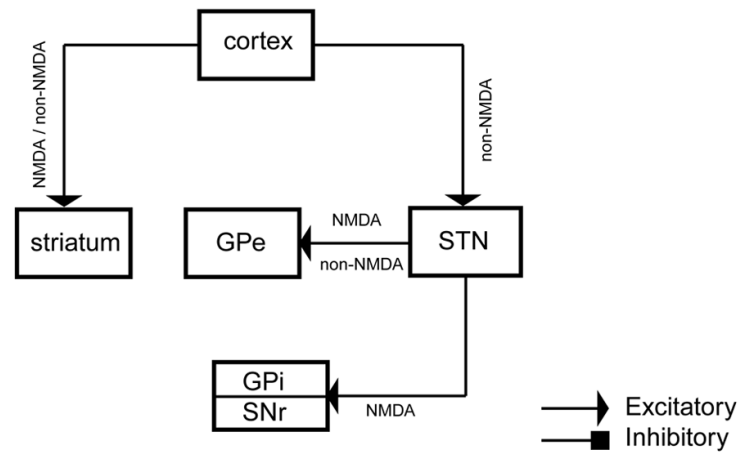

B Dopamine depletion alters cortex-BG dynamics

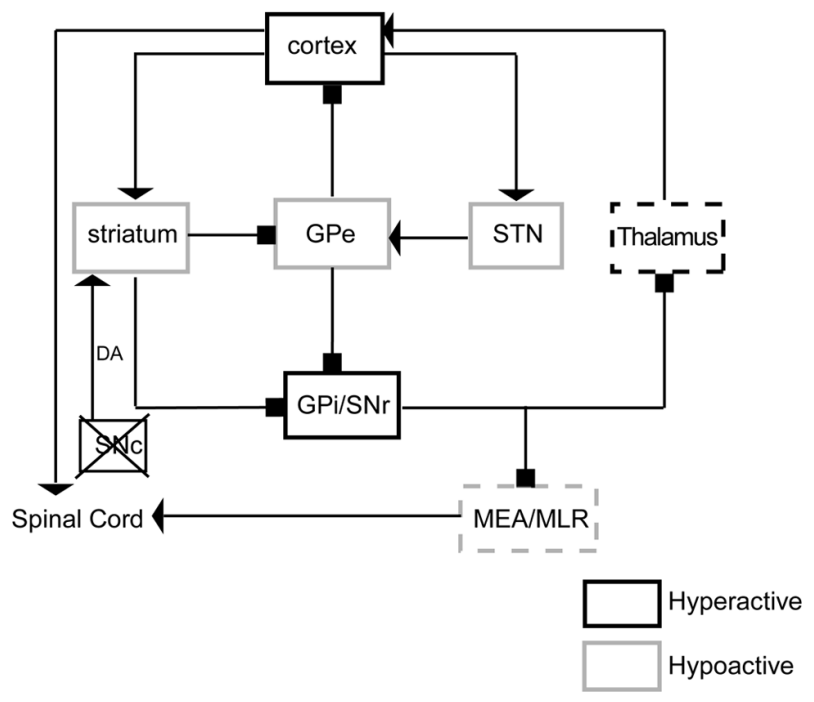

FIGURE 9 | Neurodynamic model of BG-cortex interactions. Panel A illustrates the neural pathways, neurotransmitters, and receptors by which cortex synchronizes the BG. This neural circuitry regulates BG activity. Panel $\mathbf{B}$ illustrates specific neuronal activity changes and underlying pathways in the BG network and BG-cortex circuit. DA depletions reduce activity in the striatum, $\mathrm{GPe}$, and STN and increase neuronal activity in the GPi and SNr. Hypoactive GPe causes hyperactive cortex, specifically $\mathrm{M} 1-\mathrm{M} 2$. Hyperactive GPi/SNr may reduce pontine motor control area via the midbrain extrapyramidal area (MEA) and midbrain locomotor region (MLR). The black box indicates a hyperactive region, while light boxes indicate hypoactive regions. Dashed boxes are areas that fail to show expected changes in c-Fos expression based on this model.

neurons such as pontine cholinergic neurons, midbrain dopaminergic neurons and pontine serotonergic neurons do not show robust c-Fos staining (Lu et al., 2006). On the other hand, studies in sleep-wake system, circadian and feeding regulation indicate that c-Fos in other brain regions correlate with neuronal firing activity. Notably, all brain areas in our study are able to express c-Fos, which suggests that c-Fos in those areas reflects neuronal activity. And, importantly, we examined the contrast in c-Fos expression in the same area between conditions.
BG subunits are more active (firing rates) during cortically active states like wake and REM sleep (Magill et al., 2000; Urbain et al., 2000; Lee et al., 2001). Even during sleep, striatal, GPe and STN firing activity all follow up and down states of slow oscillatory cortical activity (Magill et al., 2000; Urbain et al., 2000; Lee et al., 2001; Kasanetz et al., 2002; Mahon et al., 2006). The responses of $\mathrm{BG}(\mathrm{CPu}, \mathrm{GPe}, \mathrm{GPi}, \mathrm{STN}$, and $\mathrm{SNr}$ ) neurons to a single cortical stimulation exhibit a sequential activity pattern of excitation-inhibition-excitation (Kita and Kita, 2011). The first excitation of BG neurons results from direct cortical projections to the striatum and STN, which then stimulates GPi, GPe, and $\mathrm{SNr}$ (Tachibana et al., 2008). The subsequent inhibition and excitation are likely mediated by intra-BG interactions (Afsharpour, 1985; Rouzaire-Dubois and Scarnati, 1985). While BG and cortex appear synchronized, it was unclear whether activity of BG subunits depended on active cortical input or if subcortical input was sufficient to drive BG activity. Systemic atropine administration produced a dissociative state of cortical inhibition, subcortical activation, and BG inhibition, suggesting that the cortical inputs, rather than subcortical inputs, are required and necessary to drive overall BG activity. Subcortical inputs to the BG, including midbrain dopaminergic, pontine serotonergic and thalamic glutamatergic projections, may modulate BG activity during cortical active states. These results suggest that the BG activity is driven by cortical activity. Moreover, without active cortical inputs to the BG, such as during sleep or anesthesia, dopamine has a minimal role in modulating $B G$ activity.

Aberrant or diminished cortical activity, such as those observed in the motor cortex in MPTP- treated primates, is thought to produce Parkinsonian symptoms (Goldberg et al., 2002; Johnson et al., 2009; Pasquereau and Turner, 2011). However, 6-OHDA lesions of SNc in rats increase firing rates of the prefrontal pyramidal neurons in vitro (Zhang et al., 2011) and in vivo (Fan et al., 2011), which is consistent with our observation of high Fos expression in the motor cortex after 6-OHDA lesion. We also observed diminished striatal, GPe, and STN activity, along with hyperactivity in the GPi and SNr with dopamine depletion during active wake. The GPi/SNr hyperactivity may be due to loss of D1 action in the striatonigral neurons and/or hypoactive GPe, while hypoactivity in the STN may be caused by loss of direct dopamine D5 receptor action in the STN neurons (Ni et al., 2001; Baufreton et al., 2003). The BG can access cortex (Figure 9B) via the GPe directly (Parent and De Bellefeuille, 1983; Saper, 1984; Haber et al., 1985; Hur and Zaborszky, 2005; Vetrivelan et al., 2010), or indirectly through the mediodorsal thalamic nucleus (MD) (Haber et al., 1985). In addition, the motor thalamus relays SNr/GPi signals to the cortex. Hyperactivity of the GPi/SNr may influence motor cortex via this pathway, resulting in dyskinesia, postural instability, and difficulty turning the body in Parkinsonism. We could not detect c-Fos expression changes in the motor thalamus in the dopamine depletion condition, suggesting that GPi/SNr hyperactivity produces a subtle alteration of firing patterns rather than complete silencing of activity to influence thalamocortical projections.

In summary, our data supports the notion that cortical activity but not subcortical activity is required for BG activation. Specific 
glutamate receptors are involved in cortical control of the BG, as well as the interaction of BG subunits and ultimately BG output back to cortex. By regulating BG activity during active states, dopamine influences cortical activity to affect sleep-wake and motor behavior.

\section{ACKNOWLEDGMENTS}

This study was supported by the NIH (NS06184, NS062727), National Natural Science Foundation of China (31171049, 31121061, 31271164), Shanghai Committee of Science and Technology (11ZR1401800, 13140903100, 13dz2260700), SRF for ROCS, SEM., National Basic Research Program of China (2011CB711000, 2009ZX09303-006), and by Shanghai Leading Academic Discipline Project (B119).

\section{REFERENCES}

Abedi, P. M., Delaville, C., De Deurwaerdere, P., Benjelloun, W., and Benazzouz, A. (2013). Intrapallidal administration of 6-hydroxydopamine mimics in large part the electrophysiological and behavioral consequences of major dopamine depletion in the rat. Neuroscience 236, 289-297. doi: 10.1016/j.neuroscience.2013.01.043

Afsharpour, S. (1985). Topographical projections of the cerebral cortex to the subthalamic nucleus. J. Comp. Neurol. 236, 14-28. doi: 10.1002/cne.902360103

Baufreton, J., Garret, M., Rivera, A., De La Calle, A., Gonon, F., Dufy, B., et al. (2003). D5 (not D1) dopamine receptors potentiate burst-firing in neurons of the subthalamic nucleus by modulating an L-type calcium conductance. J. Neurosci. $23,816-825$.

Davis, C. J., Clinton, J. M., Jewett, K. A., Zielinski, M. R., and Krueger, J. M. (2011). Delta wave power: an independent sleep phenotype or epiphenomenon? J. Clin. Sleep Med. 7, S16-S18. doi: 10.5664/JCSM.1346

Detari, L., Juhasz, G., and Kukorelli, T. (1987). Neuronal firing in the pallidal region: firing patterns during sleep-wakefulness cycle in cats. Electroencephalogr. Clin. Neurophysiol. 67, 159-166. doi: 10.1016/0013-4694(87) 90039-3

Fan, L. L., Zhang, Q. J., Liu, J., Feng, J., Gui, Z. H., Ali, U., et al. (2011). In vivo effect of 5-HT(7) receptor agonist on pyramidal neurons in medial frontal cortex of normal and 6-hydroxydopamine-lesioned rats: an electrophysiological study. Neuroscience 190, 328-338. doi: 10.1016/j.neuroscience.2011.06.011

Goldberg, J. A., Boraud, T., Maraton, S., Haber, S. N., Vaadia, E., and Bergman, H. (2002). Enhanced synchrony among primary motor cortex neurons in the 1-methyl-4-phenyl-1,2,3,6-tetrahydropyridine primate model of Parkinson's disease. J. Neurosci. 22, 4639-4653.

Gompf, H. S., Mathai, C., Fuller, P. M., Wood, D. A., Pedersen, N. P., Saper, C. B., etal. (2010). Locus ceruleus and anterior cingulate cortex sustain wakefulness in a novel environment. J. Neurosci. 30, 14543-14551. doi: 10.1523/JNEUROSCI.3037-10.2010

Haber, S. N., Groenewegen, H. J., Grove, E. A., and Nauta, W. J. (1985). Efferent connections of the ventral pallidum: evidence of a dual striato pallidofugal pathway. J. Comp. Neurol. 235, 322-335. doi: 10.1002/cne.902350304

Hur, E. E., and Zaborszky, L. (2005). Vglut2 afferents to the medial prefrontal and primary somatosensory cortices: a combined retrograde tracing in situ hybridization study [corrected]. J. Comp. Neurol. 483, 351-373. doi: 10.1002/cne.20444

Irmis, F. (1971). Dissociation between EEG and spontaneous behaviour of rats after atropine. Act. Nerv. Super. (Praha) 13, 217-218.

Johnson, M. D., Vitek, J. L., and Mcintyre, C. C. (2009). Pallidal stimulation that improves parkinsonian motor symptoms also modulates neuronal firing patterns in primary motor cortex in the MPTP-treated monkey. Exp. Neurol. 219, 359-362. doi: 10.1016/j.expneurol.2009.04.022

Kasanetz, F., Riquelme, L. A., and Murer, M. G. (2002). Disruption of the twostate membrane potential of striatal neurones during cortical desynchronisation in anaesthetised rats. J. Physiol. 543, 577-589. doi: 10.1113/jphysiol.2002.00 24786

Kita, H., and Kita, T. (2011). Cortical stimulation evokes abnormal responses in the dopamine-depleted rat basal ganglia. J. Neurosci. 31, 10311-10322. doi: 10.1523/JNEUROSCI.0915-11.2011
Lee, R. S., Steffensen, S. C., and Henriksen, S. J. (2001). Discharge profiles of ventral tegmental area GABA neurons during movement, anesthesia, and the sleep-wake cycle. J. Neurosci. 21, 1757-1766.

Lu, J., and Greco, M. A. (2006). Sleep circuitry and the hypnotic mechanism of GABAA drugs. J. Clin. Sleep Med. 2, S19-S26.

$\mathrm{Lu}$, J., Jhou, T. C., and Saper, C. B. (2006). Identification of wake-active dopaminergic neurons in the ventral periaqueductal gray matter. J. Neurosci. 26, 193-202. doi: 10.1523/JNEUROSCI.2244-05.2006

Lu, J., Nelson, L. E., Franks, N., Maze, M., Chamberlin, N. L., and Saper, C. B. (2008). Role of endogenous sleep-wake and analgesic systems in anesthesia. J. Comp. Neurol. 508, 648-662. doi: 10.1002/cne.21685

Magill, P. J., Bolam, J. P., and Bevan, M. D. (2000). Relationship of activity in the subthalamic nucleus-globus pallidus network to cortical electroencephalogram. J. Neurosci. 20, 820-833.

Mahon, S., Vautrelle, N., Pezard, L., Slaght, S. J., Deniau, J. M., Chouvet, G., et al. (2006). Distinct patterns of striatal medium spiny neuron activity during the natural sleep-wake cycle. J. Neurosci. 26, 12587-12595. doi: 10.1523/JNEUROSCI.3987-06.2006

Nauta, H. J. (1979). Projections of the pallidal complex: an autoradiographic study in the cat. Neuroscience 4, 1853-1873. doi: 10.1016/0306-4522(79) 90060-5

Ni, Z., Gao, D., Bouali-Benazzouz, R., Benabid, A. L., and Benazzouz, A. (2001). Effect of microiontophoretic application of dopamine on subthalamic nucleus neuronal activity in normal rats and in rats with unilateral lesion of the nigrostriatal pathway. Eur. J. Neurosci. 14, 373-381. doi: 10.1046/j.0953-816x.2001. 01644.x

Parent, A., and De Bellefeuille, L. (1983). The pallidointralaminar and pallidonigral projections in primate as studied by retrograde double-labeling method. Brain Res. 278, 11-27. doi: 10.1016/0006-8993(83)90222-6

Pasquereau, B., and Turner, R. S. (2011). Primary motor cortex of the parkinsonian monkey: differential effects on the spontaneous activity of pyramidal tract-type neurons. Cereb. Cortex 21, 1362-1378. doi: 10.1093/cercor/ bhq217

Qiu, M. H., Vetrivelan, R., Fuller, P. M., and Lu, J. (2010). Basal ganglia control of sleep-wake behavior and cortical activation. Eur. J. Neurosci. 31, 499-507. doi: 10.1111/j.1460-9568.2009.07062.x

Ravenscroft, P., and Brotchie, J. (2000). NMDA receptors in the basal ganglia. J. Anat. 196, 577-585. doi: 10.1046/j.1469-7580.2000.19640577.x

Rouzaire-Dubois, B., and Scarnati, E. (1985). Bilateral corticosubthalamic nucleus projections: an electrophysiological study in rats with chronic cerebral lesions. Neuroscience 15, 69-79. doi: 10.1016/0306-4522(85)90124-1

Saper, C. B. (1984). Organization of cerebral cortical afferent systems in the rat. II. Magnocellular basal nucleus. J. Comp. Neurol. 222, 313-342. doi: $10.1002 /$ cne. 902220302

Schaul, N., Gloor, P., Ball, G., and Gotman, J. (1978). The electromicrophysiology of delta waves induced by systemic atropine. Brain Res. 143, 475-486. doi: 10.1016/0006-8993(78)90358-X

Tachibana, Y., Kita, H., Chiken, S., Takada, M., and Nambu, A. (2008). Motor cortical control of internal pallidal activity through glutamatergic and GABAergic inputs in awake monkeys. Eur. J. Neurosci. 27, 238-253. doi: 10.1111/j.14609568.2007.05990.x

Urbain, N., Gervasoni, D., Souliere, F., Lobo, L., Rentero, N., Windels, F., et al. (2000). Unrelated course of subthalamic nucleus and globus pallidus neuronal activities across vigilance states in the rat. Eur. J. Neurosci. 12, 3361-3374. doi: 10.1046/j.1460-9568.2000.00199.x

Vetrivelan, R., Fuller, P. M., Tong, Q., and Lu, J. (2009). Medullary circuitry regulating rapid eye movement sleep and motor atonia. J. Neurosci. 29, 9361-9369. doi: 10.1523/JNEUROSCI.0737-09.2009

Vetrivelan, R., Qiu, M. H., Chang, C., and Lu, J. (2010). Role of Basal Ganglia in sleep-wake regulation: neural circuitry and clinical significance. Front. Neuroanat. 4:145. doi: $10.3389 /$ fnana.2010.00145

Yelnik, J. (2008). Modeling the organization of the basal ganglia. Rev. Neurol. (Paris) 164, 969-976. doi: 10.1016/j.neurol.2008.04.019

Zhang, Q. J., Li, L. B., Niu, X. L., Liu, J., Gui, Z. H., Feng, J. J., etal. (2011). The pyramidal neurons in the medial prefrontal cortex show decreased response to 5-hydroxytryptamine- 3 receptor stimulation in a rodent model of Parkinson's disease. Brain Res. 1384, 69-79. doi: 10.1016/j.brainres.2011. 01.086 
Conflict of Interest Statement: The authors declare that the research was conducted in the absence of any commercial or financial relationships that could be construed as a potential conflict of interest.

Received: 21 January 2014; accepted: 03 March 2014; published online: 26 March 2014 Citation: Qiu MH, Chen MC, Huang ZL and Lu J (2014) Neuronal activity (c-Fos) delineating interactions of the cerebral cortex and basal ganglia. Front. Neuroanat. 8:13. doi: 10.3389/fnana.2014.00013
This article was submitted to the journal Frontiers in Neuroanatomy.

Copyright (C) 2014 Qiu, Chen, Huang and Lu. This is an open-access article distributed under the terms of the Creative Commons Attribution License (CC BY).

The use, distribution or reproduction in other forums is permitted, provided the original author(s) or licensor are credited and that the original publication in this journal is cited, in accordance with accepted academic practice. No use, distribution or reproduction is permitted which does not comply with these terms. 